Keio J. Med. 22: 163-171, 1973

\title{
THE DEGENERATING PATTERN OF THE MONKEY PYRAMIDAL TRACT FIBERS FOLLOWING THE VARIOUS CORTICAL LESIONS: INCLUDING THE CORTICOBULBAR AND CORTICOCEREBELLAR TRACT
}

\author{
PART 1. THE QUALITATIVE STUDY
}

\author{
HIROKAZU NAITO \\ Division of Neurosurgery, Department of Surgery, School of Medicine, \\ Keio University, Tokyo, Japan
}

(Received for publication November 12, 1973)

\begin{abstract}
Following the various lesions in the cerebral cortex of the monkeys, the degenerated fibers were traced in the level of the cerebral peduncle, pons and pyramid of the medulla oblongata. Extensive intermingling of the projection fields is demonstrated at the cerebral peduncle but there is a denser aggregation of the face fibers toward the medial side, arm fibers in the middle and foot fibers toward the lateral side of the middle $3 / 5$ of the cerebral peduncle.

The parietopontine tract passes at the lateral $2 / 5$ of the pes pedunculi. In the pons, the parietopontine tract is rather concentrated in the dorso-lateral groups of the longitudinally descending bundles. Otherwise further extensive intermingling of the fibers occurs in the pontine level and no clear cut somatotopographical pattern is found.

In the medullary pyramid, the degenerated fibers are diffusely and homogenously distributed. Small numbers of fibers in the motor face lesion still descend beyond the medullary pyramid. It was found that the pyramidal tract fibers were not clearly demarcated topographically in the brain stem as described in the diagram of some literatures.
\end{abstract}

\section{INTRODUCTION}

Since the earlier era of the modern neurology, the prominent medullary pyramid was considered to be the main pathway of the motor system and has been extensively studied in both human beings and animals..$^{1-0}$

The somatotopographical arrangement of fibers within the pyramidal tract 
at different levels of the neuroaxis has been one of the intriguing subjects. Foerster illustrated the diagram of the spinal cord with leg fibers laterally and arm fibers medially. ${ }^{10}$ Although this has never been witnessed anatomically, many accepted and used this diagram in the daily practice until recently. Concerning the topography in the cerebrum, Penfield and Rasmussen completed the closely woven homunculus of the motor cortex. ${ }^{11}$ This knowledge came from the painstaking electrical stimulation of the cerebral cortex in numerous conscious patients. Since Jacksonian seizure marches in the order of the topography and paralysis by the lesion of the motor cortex correlates with Penfield's homunculus, the topographical organization of the motor neurons in the cerebral cortex undoubtedly exists. Similar topographical arrangement in the monkey cortex was established by Fulton, Dusser de Barenne and Woolsey.12-14

Once cortically originating pyramidal tract fibers descend and aggregate in the deep narrow internal capsule, the topographical pattern becomes increasingly difficult subject to investigate. As long as the axons separated from the cells inevitably degenerate, the cortical ablation in the monkey provides the tracing of the descending fibers in the different levels of the neuroaxis. This series of operations was intended to study the degenerating fiber patterns in the brain stem and to count the preserved fibers in the medullary pyramid. The author describes here the former subject as Part 1-Qualitative study.

\section{MATERIALS AND METHODS}

A total of 14 monkeys, Macaca mulatta, was used for this study. Four of 14 brains were discarded because of their inadequate postoperative survivals. The monkeys were anesthetized with the intramuscular injection of Ketalar $(5 \mathrm{mg} / \mathrm{kg}$ ) followed by the intravenous injection of Nembutal $(25 \mathrm{mg} / \mathrm{kg})$. Using the maps of Fulton's monograph for guidance, the craniectomy was performed over the intended lesion (Fig. 1). The cortex to be excised was identified by the characteristic pattern of the cerebral sulci and was marked by the cautery. The cortical incision was made down to the bottom of the sulci and the intended region was removed by a gentle suction. All the lesions were made in the left hemisphere and each monkey was subjected to one of these following operations: 1 ) hemispherectomy, 2) hemicortectomy, 3) decortication of the precentral and postcentral gyrus, 4) frontal lobectomy, 5) parietal lobectomy, 6) decortication of the precentral gyrus, 7) decortication of the upper $1 / 3$ of the precentral gyrus, 8) decortication of the middle $1 / 3$ of the precentral gyrus and 9 ) decortication of the lower $1 / 3$ of the precentral gyrus. Except hemispherectomy, the main arterial 
trunks were preserved.

Basal ganglia and thalamus were intact in hemispherectomy but the large part of the caudate nucleus was removed.

In hemicortectomy, the cortex of the convexity was removed down to the bottom of the cerebral sulci but the subcortical nuclei were left in place. In frontal lobectomy, the cingulate gyrus anterior to the central sulcus was removed. Bleeding was controlled by gelfoam or cautery.

The postoperative functional deficit was examined and assessed (Fig. 2). Except the monkey of decortication of lower $1 / 3$ of the precentral gyrus, all showed slight to severe hemiparesis. The photography as well as movie were taken in some of these monkeys before the sacrifice. The hemispherectomied monkey died 15 days after the operation. Other monkeys were killed at the interval between 6 weeks and 17 weeks after the operation. Under the similar anesthesia with the preceding operation, the intravital perfusion with saline and $10 \%$ formalin was performed by catheterization into the ascending aorta. The whole

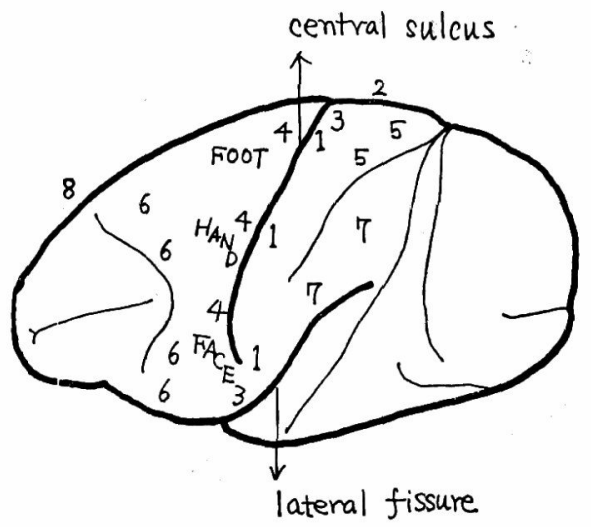

Fig. 1 The fissural pattern of the lateral surface of the monkey cerebral cortex. The area was designated by the Brodmann's number.

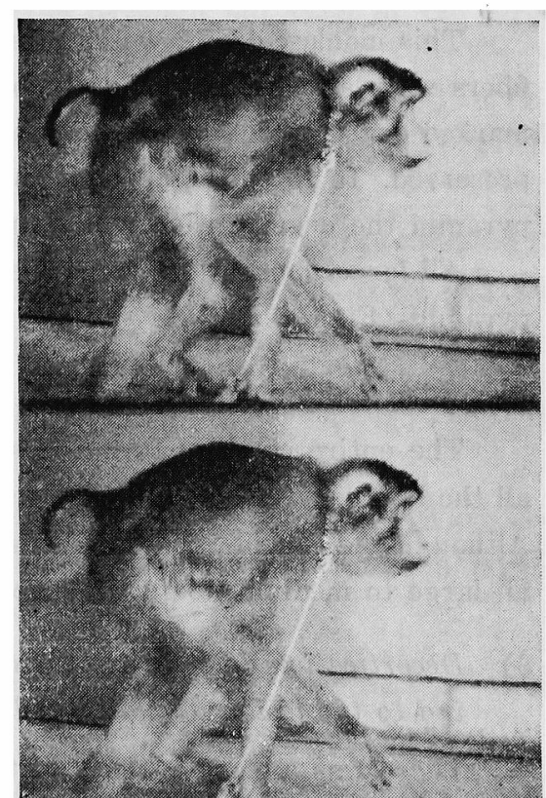

Fig. 2 This photo was taken 3 months after the decortication of the left hemisphere. See the flexor posture of the right arm and slow gait with favoring the right leg. 
neuroaxis was dissected out and fixed in $10 \%$ formalin for more than 2 weeks for the histological study. The extent of the cerebral lesion was photographed.

The transverse section of the midbrain, pons and medullary pyramid was submitted to the microscopic study.

The following staining methods were used for tracing the preserved and degenerated fibers. Normal myelin is stained blue but degenerated myelin is unstained by luxol fast blue overstained with hematoxylin eosin. Bodian's method impregnates neurofiber by silver. The degenerated myelin eventually changes into lipids which can be stained by oil red $O$ as red or orange. Nauta-Gygax method stains the small degenerated nerve fibers as black. Toluidine blue stain after paraffin fixation is used for seeing further details of axons. The extent and severeness of the degeneration were assessed by the combination of the review of these slides.

\section{RESULT}

\section{1) Hemispherectomy}

This monkey died 2 weeks after the operation. Therefore, many small nerve fibers were still preserved in the brain stem. The cerebral peduncle showed the marked degeneration but the medial $1 / 5$ and the lateral $2 / 5$ of it were relatively preserved. In the pons, all bundles were degenerated in equal degree and in the pyramid the degeneration was diffuse.

All large fibers were under the process of the degeneration in the medullary pyramid (Figs. 3 and 4 ).

\section{2) Hemicortectomy}

The entire cerebral peduncle showed the severe degeneration. In the pons, all the bundles were equally degenerated. Entire pyramid degenerated markedly. Although the considerable numbers of the small fibers still remain intact, almost all large to medium fibers were gone.

3) Decortication of both precentral and postcentral cortex; roughly corresponding to the lesion of motor and sensory cortex

There was a clear sparing of the degeneration at the medial $1 / 5$ of the pes and moderate sparing at the lateral $1 / 3$ of the pes. All bundles in the pons were degenerated but the peripherally locating bundles seemed to be less affected.

In the pyramid, the degeneration was diffuse and homogenous. 


\section{4) Frontal lobectomy}

There was a definite preservation of the nerve fibers at the lateral $1 / 3$ of the pes. The dorsolaterally locating bundles in the pons showed less degeneration. In the pyramid, the degeneration was throughout with equal degree.

\section{5) Decortication of the precentral gyrus}

There was a relative sparing of the degeneration at the medial $1 / 5$ and the lateral $1 / 3$ of the pes. All longitudinal bundles seemed to be equally degenerated in the pons. The entire pyramid was degenerated with the preservation of the small fibers.

\section{6) Parietal lobectomy}

The well demarcated degeneration was seen in the lateral 2/5 of the pes which represented the parietopontine tract (Fig. 5). The degeneration was seen in the adjacent middle $2 / 5$ of the pes but the medial $1 / 5$ of the pes was preserved. In the pons, the laterodorsally locating bundles were rather heavily degenerated. Minimal diffuse degeneration was noted in the pyramid but most of the large fibers were preserved.

7) Decortication of the upper $1 / 3$ of the precentral gyrus; roughly corresponding to the motor foot lesion

The degeneration was most concentrated in the lateral part of the middle $1 / 3$ of the pes. In the pons, the degeneration was scattered in all bundles and the pyramid showed the equal distribution of the degenerated fibers (Fig. 6).

8) Decortication of the middle $1 / 3$ of the precentral gyrus; roughly equivalent to the motor hand lesion

The degeneration was seen in the middle $1 / 3$ of the pes. In the pons, the degeneration was diffuse in all bundles. Medullary pyramid showed a homogenously distributed degeneration with no particular aggregation.

9) Decortication of the lower $1 / 3$ of the precentral gyrus; roughly equivalent to the motor face lesion

The degenerated fibers were clustered in the medial portion of the middle $1 / 3$ of the pes. In the pons, the slight degeneration with no particular concentration of the degenerated fibers was noted. In the pyramid, the degenerated fibers were still sparsely and diffusely present. 


\section{DISCUSSION}

The pyramidal tract along with the corticobulbar and corticocerebellar tract forms the fairly discrete pathway in the pes pedunculi of the midbrain. The pyramidal tract divides into multiple bundles which interdigitate with the nuclei and fibers of the pons but again aggregate into the pyramid on the ventral surface of the medulla oblongata. Most of these fibers decussate at the transitional level to the spinal cord. This pathway traced by the nerve fiber degeneration is a main motor system. Other indistinct pathway known as an extrapyramidal tract is not the scope in this study and there is no further reference here.

The somatotopographical arrangement of the fibers within the pyramidal tract at the brain stem is still controversial subject. It exists undoubtedly in the cerebral cortex with the complicated overlapping pattern. At the internal capsule, further intermingling of fibers occurs. In the cerebral peduncle, the topographical arrangement is still preserved. The parietopontine tract originating from the parietal lobe is lateral most in the peduncle. Medial to this, the pyramidal tract fibers from the precentral gyrus are arranged in an orderly fashion with marked intermingling. The foot fibers laterally, hand fibers in the middle and the face fibers medially with the extensive overlap. The most medial $1 / 5$ of the pes pedunculi is relatively preserved in the decortication of the precentral gyrus. In the pons, more parietopontine tract fibers occupy the dorsolateral bundles but the overlapping is so extensive that no clear topographical arrangement can be documented. In contrast to the diagram in the neurological literature, the author does not find any clear topographical arrangement of the pyramidal tract fibers in the pyramid of the monkey.

This result is consistent with Tower's experiences. ${ }^{15}$ She made 3 different types of partial section of the medullary pyramid in 3 groups of monkeys and observed the similar functional defect in each groups. As long as these observations are concerned, the commonly used illustration of the laminated somatotopographical pattern in the pyramid of man; the foot fibers laterally, the trunk fibers in the middle and the hand fibers medially, needs further investigations.

\section{SUMMARY}

Following the various cortical lesions in the monkeys, the degenerating fibers of the pyramidal tract were traced by the several staining methods. There is a somatotopographical arrangement of the fibers in the cerebral peduncle.

However, there is no such arrangement of fibers in the pons or medullary pyramid. 


\section{Acknowledgement}

The author appreciates the kind help and advices of Dr. T. Hasegawa, Dr. S. Toya, Dr. T. Sasaki, Dr. Y. Shigematsu, Miss E. Kusakari and Miss M. Shirasawa. The microscopic study was kindly prepared by Dr. H. Matsuyama, Dr. K. Ogawa and Dr. Y. Inoue.

\section{REFERENCES}

1. Holmes, E. G. and May, W. P.: On the exact origin of the pyramidal tract in man and other mammals. Brain 32: 1-43, 1909

2. Monakow, C. V.: Zur Anatomie und Physiologie der Pyramidenbahn und der Armregion, nebst Bemerkungen über die sekundäre Degeneration des Fasciculus centroparietalis. Neurol. Centralbl. 34: 217-224, 1915

3. Lassek, A. M.: The pyramidal tract of monkey: A Betz cell and pyramidal tract enumeration. J. Comp. Neurol. 74: 193-202, 1941

4. Bucy, P. C.: Is there a pyramidal tract? Brain 80: 376-392, 1957

5. Bucy, P. C.: Effffects of extirpation in man. In: The precentral motor cortex. 2nd ed. edited by Bucy, P. C., University of Illinois Press, Urbana, 1949, 353-394

6. Bucy, P. C., Keplinger, J. E. and Siqueira, E. B.: Destruction of "pyramidal tract" in man. J. Neurosurg. 21: 385-398, 1964

7. Walker A. E. and Richter, H.: Section of the cerebral peduncle in the monkey. Arch. Neurol. 14: 231-240, 1966

8. Tower, S. S.: Pyramidal lesion in the monkey. Brain 63: 36-90, 1940

9. Russell, J. R. and DeMyer, W.: The quantitative origin of pyramidal axons of Macaca rhesus, with some remarks on the slow rate of axolysis. Neurology. 11: $96-108,1961$

10. Foerster, O.: Symptomatologie der Erkrankungen des Rückenmarks und seiner Wurzeln. In: Handbuch der Neurologie, Vol. 5, edited by Bumke and Foerster, 0. Springer, Berlin, 1936, 1 -403

11. Penfield, W. and Rasmussen, T.: The cerebral cortex of man, New York, The Macmillan Co., 1950, 1-248

12. Fulton, J. F.: Physiologie des lobes frontaux et du cervelet. 1st ed., Masson et Cie, Paris, 1953, 24-55

13. Dusser de Barenne, J. G.: "Corticalization" of function and functional localization in the cerebral cortex. Arch. Neurol. \& Psychiat. 30: 884-901, 1933

14. Woolsey, G. N. and Fairman, D.: Contralateral, ipsilateral and bilateral representation of cutaneous receptors in somatic areas I and II of the cerebral cortex of pig, sheep, and others. Surgery 19:684-702, 1946

15. Tower, S.: The pyramidal tract. In: The precentral motor cortex. 2 nd ed., edited by Bucy, P. C., University of Illinois Press, Urbana, 1949, 157 


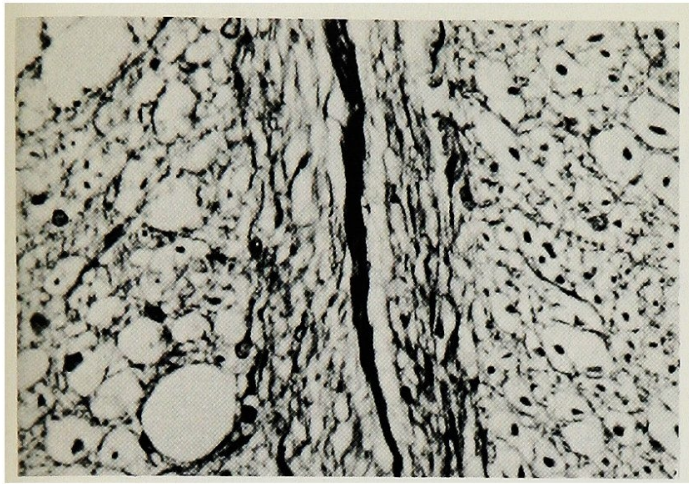

Fig. 3

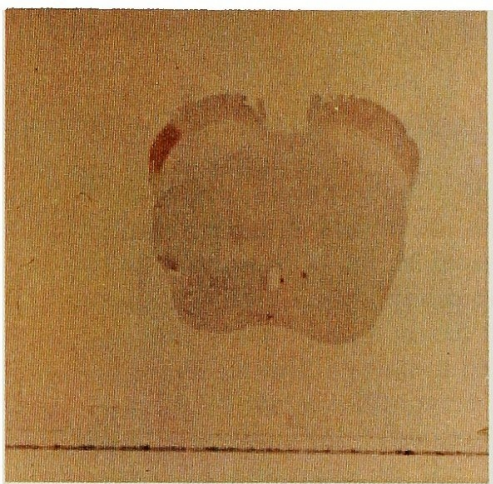

Fig. 5

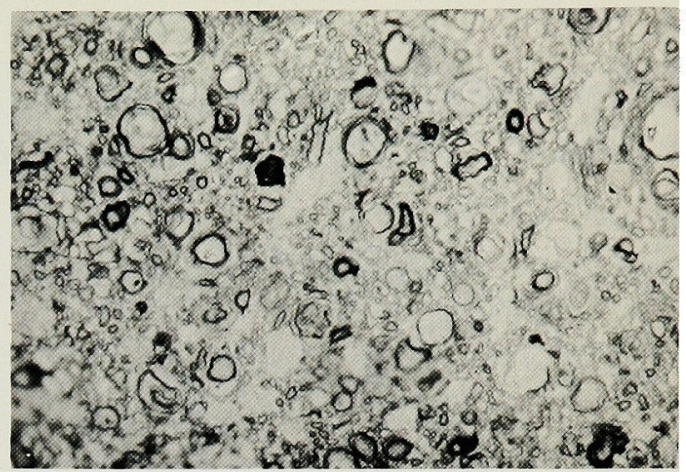

Fig. 4

Fig. 3 See the normal right pyramid in contrast to vacuolized and loose left pyramid under the degeneration. Note also the increased glia cells in the left pyramid. Bodian stain. $\times 400$

Fig. 4 All medium to large myelinated fibers are under the degenerating process. See the marked swelling of these axons. Also note the preservation of the small fibers. T.B. Stain. $\times 400$

Fig. 5 See the well demarcated positive fat stain of the lateral $2 / 5$ of the left cerebral peduncle. Oil red 0 stain. $\times 9$

Fig. 6 See the diffuse and homogenous demyelination in the left pyramid in spite of the motor foot lesion. Compare with the normal right pyramid. L.F.B.H.E. $\times 40$

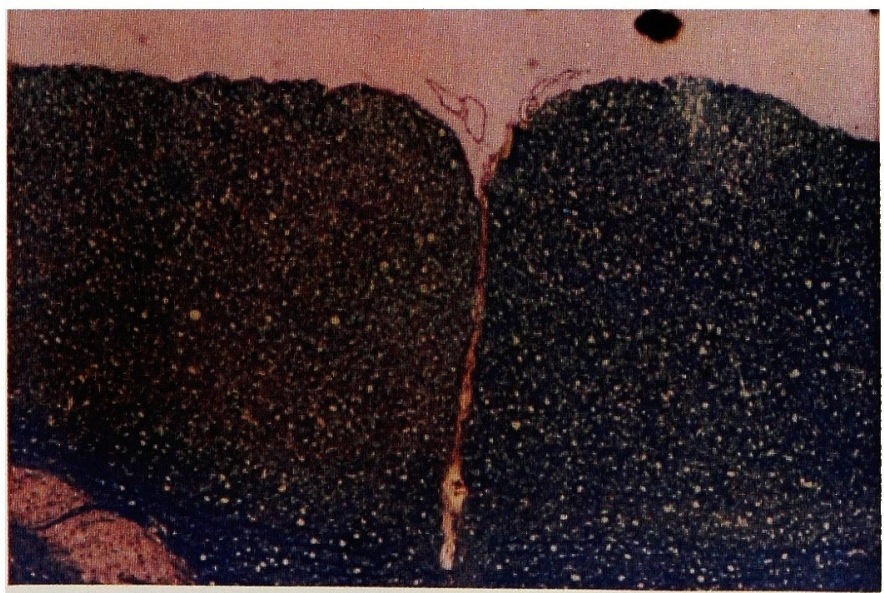

Fig. 6 